Marquette University

e-Publications@Marquette

College of Education Faculty Research and

Publications

Education, College of

$10-1-2018$

\title{
Implications for Wellness-Based Supervision and Professional Quality of Life
}

Karisse A. Callender

Marquette University, karisse.callender@marquette.edu

A. Stephen Lenz

Texas AઐM University - Corpus Christi

Accepted version. Journal of Counseling \& Development, Vol. 96, No. 4 (October 2018): 436-448.

DOI. (C) 2018 John Wiley \& Sons, Inc. Used with permission. 


\title{
Marquette University
}

\section{e-Publications@Marquette}

\section{Education Faculty Research and Publications/College of Education}

\author{
This paper is NOT THE PUBLISHED VERSION; but the author's final, peer-reviewed \\ manuscript. The published version may be accessed by following the link in the citation below.
}

Journal of Counseling and Development, Vol. 96, No. 4 (September 11, 2018): 436-448. DOI. This article is (C) American Counseling Association and permission has been granted for this version to appear in e-Publications@Marquette. American Counseling Association does not grant permission for this article to be further copied/distributed or hosted elsewhere without the express permission from American Counseling Association.

\section{Implications for Wellness-Based Supervision and Professional Quality of Life}

Karisse A. Callender

Department of Counselor Education and Counseling Psychology, Marquette University and Stephen Lenz

Department of Counseling and Educational Psychology, Texas A\&M University - Corpus Christi.

This research was funded by the Association for Counselor Education and Supervision (ACES)

Research Grant. Correspondence concerning this article should be addressed to Karisse A. Callender, Department of Counselor Education and Counseling Psychology, 561 N. 15 ${ }^{\text {th }}$ Street, SC168G, Milwaukee, WI, 53233 (e-mail: karissecallender@gmail.com).

\footnotetext{
Abstract

This study evaluated the efficacy of the Wellness Model of Supervision (WELMS; Lenz \& Smith, 2010) for promoting changes across counselors-in-training perceptions of professional quality of life. Participants were three women who identified as Caucasian $(n=1)$ and Hispanic $(n=2)$, enrolled in a CACREP accredited program. Results of a single case research design (SCRD) with multiple baselines indicated the WELMS was efficacious across client-CIT interactions on professional quality of life.
}

\section{Keywords}

Wellness, Supervision, Single-Case Research Design, Professional Quality of Life 
Supervision of counselors-in-training (CITs) is a signature pedagogy within counselor preparation programs that promotes skill development, monitors client welfare, and assesses students' fitness to practice using structured and unstructured interventions to (Bernard \& Goodyear, 2014; Goodyear, Bunch, \& Claiborn, 2006). Whereas CITs spend a considerable proportion of their degree programs in classroom-based, didactic learning environments, field experiences provide community-based, experiential educational venues for implementing counseling skills with client populations. Supervision processes are developmentally supportive during this time, while also fostering professional identity through monitoring interventions that promote positive client outcomes. Borders et al. (2014) recommended several evidence-based best practices for supervisors to implement for promoting CIT self-evaluation and reflection. One of those best practices situates counseling supervisors as gatekeepers for the profession by monitoring the development, functioning, and wellness of CITs; qualities indicative of fitness to practice. Taken together, the most effective approaches to CIT supervision will contribute to client welfare by not only developing clinical skills, but also promoting student career sustaining behaviors.

The importance of this supposition is reflected in the American Counseling Association Code of Ethics (ACA; 2014), which stipulates counselors engage in self-care activities that monitor and maintain their well-being as part of their professional responsibilities. Counselors are therefore encouraged to monitor two vital relationships: between their personal well-being and therapeutic effectiveness, and the relationship between counselors and their colleagues. One construct used as a proxy for the coalescence of these three relationships is professional quality of life (Lawson \& Myers, 2011). Professional quality of life (ProQOL) refers to the value of the work done in relation to the individual's role as a counselor-in-training which includes compassion satisfaction and compassion fatigue (Stamm, 2010). When CITs are exposed to triggers with the potential to diminish their mental and physical resources, their ProQOL decreases. A lack of compassion and burnout, due to a decreased professional quality of life, may lead to feelings of anxiety, depression, and helplessness in CITs (Perkins \& Sprang, 2013).

By contrast, when professional quality of life, and therefore wellness, are positively influenced, CITs have well-developed traits of interpersonal and intrapersonal resources to mitigate stress levels, and CITs with these attributes, who are actively working toward a state of wellness, may increase their effectiveness as counselors. In addition, having these healthy attributes may help CITs to establish a deeper level of connection with their clients and increased levels of compassion (Burck, Bruneu, Baker, \& Ellison, 2014; Neswald-Potter, Blackburn, \& Noel, 2013)

Time spent during supervision presents an opportunity to learn more about CITs’ professional development. Supervisors may have a chance to learn how CITs cope with the demands of the profession and monitor how stressors associated with counseling may affect the personal wellness of CITs. In addition, utilizing a Wellness Model of Supervision (WELMS; Lenz \& Smith, 2010) may help to avert gatekeeping issues, such as burnout and unethical behavior that may rupture career-sustaining behaviors. Therefore, a model of supervision integrating wellness monitoring and development may be essential in preventing and managing the risks associated with counseling. A wellness model of supervision may also provide an opportunity for personal and professional growth (Lawson \& Myers, 2011; Lenz \& Smith, 2010).

\section{Logic Behind WELMS for Promoting Skills Development and Personal Wellness}

During supervision, supervisors monitor CITs’ development in their knowledge and application of professional skills, and their general wellness and self-care practices for progress. Supervisors have an ethical obligation to attend to the holistic well-being and self-care of CITs (Ohrt, Prosek, Ener, \& Lindo, 2015). Therefore, supervision is conceptualized as the driving force mediating and regulating the personal and professional development of CITs, and the therapeutic intervention between CITs and client.

Thompson, Frick, and Trice-Black (2011) suggested that CITs desired supervisor support about strategies for work-life balance; however, there are currently only a few empirical studies demonstrating causal 
relationships between wellness-based supervisory interventions and their related effects on CIT wellness (Lenz, Sangganjanavanich, \& Balkin, 2012; Meany-Walen, Davis-George, \& Lindo, 2016; Storlie \& Smith, 2012). In the absence of this body of literature, it becomes difficult for counselor educators to make data driven decisions about supervisory interventions that may be most efficacious for promoting the development of clinical skills, monitoring client welfare, and assessing students' fitness to practice.

Several scholars suggested a positive relationship between CITs’ personal wellness and their professional performance (Blount \& Mullen, 2015; Lenz, Oliver, \& Sangganjanavanich, 2014; Lenz, Sangganjanavanich, Balkin, Oliver, \& Smith, 2012; Lenz \& Smith, 2010; Ohrt et al., 2015). Although CITs expend considerable effort promoting the use of wellness strategies among their clients, they may have difficulty adapting these wellness principles into their own lifestyle, leading to an increased risk to the deleterious effects of vicarious trauma that can lead to burnout.

Lenz et al. (2014) found that CITs wellness during the graduate program has considerable implications for professional and personal development. In addition, their findings suggest that the quality of the counseling experience is higher for their clients when compared to CITs who did not receive the WELMS. In a qualitative study conducted by Lenz et al. (2012), they discovered that one mechanism for increasing the self-care practices of CITs is the WELMS. The WELMS also provided a way for supervisors to facilitate continuous wellness related activities during internship when it is most applicable and practical.

The professional quality of a counselor's life (ProQOL) should reflect self-awareness skills, an appreciation for the complexity of society, and a genuine respect for human diversity. Training and preparation for a professional quality of life includes having a secure base of trust, safety, and comfort. Counselors should place an emphasis on developing relational skills to prepare them for a life of service that is confident and passionate. Emotional self-care activities may play a significant role in professional quality of life (Bloomquist, Wood, Friedmeyer-Trainor, \& Kim, 2015). The ProQOL is an important dimension to investigate as it can reflect the quality of work that CITs engage in with their clients. A study investigating the ProQOL of CITs, using a WELMS intervention is essential to assist students to identify their potential to experience burnout and compassion fatigue. In addition, a study utilizing the WELMS would help counselor educators and supervisors have a better understanding of how CITs view themselves as professionals. This study provided a structured, yet individualized, wellness model of supervision with potential to inspire change in counselors-in-training, as their professional quality of life and personal wellness are discussed and assessed in relation to their professional identity development.

\section{Purpose of the Study}

The purpose of the study was to evaluate the efficacy of the Wellness Model of Supervision (WELMS; Lenz \& Smith, 2010) for promoting desired change across CIT perceptions of professional quality of life. This variable was selected based on the logic that the outcome will concurrently promote career sustaining behaviors and positive client outcomes. This study aimed to assist counselor educators in further understanding the efficacy of wellness interventions and provide protocols for a non-standardized model of supervision which can benefit counselors-in-training in a master's program as they learn about the role of their professional quality of life with clients. We were guided by two research questions: (a) What is the course of response to intervention for selfreported compassion satisfaction, burnout, and secondary traumatic stress associated with participation in the WELMS?; (b) To what degree is the WELMS efficacious for stimulating desired treatment effect for CIT level of compassion satisfaction, burnout, and secondary traumatic stress over time?

\section{Method}

We implemented a small series A-B single-case research design (SCRD) with multiple baselines to evaluate the degree of efficacy for the WELMS in promoting professional quality of life among CITs over time. This design was selected based on the ability to use a small sample size and practicality for estimating functional relationships associated with an intervention and target outcomes (Kazdin, 2011). 


\section{Measurement of Constructs}

Professional Quality of Life. The Professional Quality of Life Scale (ProQOL; Stamm, 2010) was developed to assess the effects of working with others who may have experienced stressful events. The ProQOL is a 30-item self-report measure that yields multiple subscales. The 10-item Compassion Satisfaction subscale was intended to quantify the degree of pleasure derived from doing well with your work, using items such as "I get satisfaction from being able to help people.” Higher scores indicate higher levels of compassion satisfaction while lower scores indicate compassion fatigue. The 10-item Burnout subscale refers to the negative effects of caring for others and includes feelings of hopelessness and feeling ineffective at one's job, using items such as "I am not as productive at work because I am losing sleep over traumatic experiences of the persons I help.” Higher scores on the burnout subscale indicate the individual believes they are not making a difference in their work, they may be overwhelmed, or function in a non-supportive environment. The 10-item secondary traumatic stress subscale, which is an element of compassion fatigue and refers to negative effects caused by exposure to others who have experienced traumatic events, is measured by items such "I can't recall important parts of my work with trauma victims." Higher scores on the secondary traumatic stress subscale indicate the individual is experiencing intrusive thoughts or images, experiencing sleep difficulties, or avoiding any reminder of their client's trauma (Stamm, 2010). Stamm reported scores on the ProQOL within the good range for internal consistency for the Compassion Fatigue, Burnout, and Secondary Trauma subscales $(\alpha=.88, .75, .81$, respectively).

Wellness. The Five Factor Wellness Inventory (5F-WEL; Myers \& Sweeney, 2005, 2014) is an evidencebased assessment, used to evaluate the characteristics of wellness as a basis for helping individuals make healthier life choices. The 5F-WEL is a 91-item self-scoring tool measuring 17 discrete scales (third order factors) based on the Indivisible Self with responses ranging from strongly disagree, disagree, agree, and strongly agree with higher scores indicating greater degree of wellness. Hattie, Myers, and Sweeny (2004) reported reliability coefficients within the excellent range for 5F-WEL total wellness $(\alpha=.94)$ and the second-order factors of Creative Self $(\alpha=.93)$, Coping Self $(\alpha=.92)$, Social Self $(\alpha=.94)$, Essential Self $(\alpha=.91)$, and Physical Self $(\alpha=$ $.90)$, indicating a robust reliability of scores.

\section{Participant Characteristics}

Participants were women enrolled in a mental health counseling internship course (300 hours) in a CACREP-accredited counseling program and completing field experience requirements at their university counseling and training clinic. Two of the three participants were completing degree programs in marriage, family, and couples counseling and one was pursuing a clinical mental health counseling specialty. Two participants were in internship II and one participant in internship I. Two participants identified with a Hispanic (67\%) ethnic identity and one (33\%) identified as Caucasian.

Diane. Diane was a 50-year-old Hispanic woman, married with adult children. Diane was enrolled in Internship II, worked full time and attended school full time. She was affable, curious, motivated, and appreciated structure. She was very committed to her clients and was comfortable with micro-skills. Diane had periods of very high stress and appeared overwhelmed with her personal circumstances. Despite the external challenges, Diane persevered throughout the semester and showed resilience amid chaos. Initially, during supervision she was very guarded, did not appear very open to sharing details about her clients, and was reluctant to accept suggestions or challenge her clinical skills. As time progressed, Diane became more open and receptive to feedback and began improving her clinical skills. She had an average of 10 clients per week, primarily adults. Her clients' presenting issues included depression, anxiety, bereavement, trauma, and substance abuse. Diane identified a desire to improve her Creative Self domain of the 5F-WEL with the expectation that she would (a) "not have an ache in my stomach due to anxiety,” (b) "no racing thoughts,” (c) “won’t feel miserable with myself,” and (d) "less countertransference.” Diane was compliant with the WELMS supervision plan and reported engaging in her wellness plan as often as she could.

Susan. Susan was a 24-year old, single, Hispanic woman who attended school full time. Susan was an Internship I student and worked part-time. She displayed a gentle demeanor and was internally motivated, caring, 
and organized. Susan presented as comfortable with her clients and her role as a counselor-in-training. She was intentional about her approach and became personally invested in the progress of her clients, motivating her to explore several options in session to help her clients attain their goals. She was open to feedback while processing client conceptualization during supervision. Susan had an average of 20 clients per week ranging from children to adults. Her clients' presenting issues included depression, anxiety, trauma, sexual abuse, and adjustment disorder. Susan identified a desire to improve her Physical Self domain of the 5F-WEL with the expectation that she would (a) "have more energy," (b) "be more effective and present with clients," "would not feel like a drag at the end of the day,” and (c) “improve sleep.” Susan was compliant with the WELMS supervision plan and reported consistent engagement in her wellness activities.

Mary. Mary was a 49-year old, married, Caucasian woman with adult children who was enrolled in Internship II, and attended school fulltime. She was very organized, internally motivated, and eager to learn new skills. She used humor to connect with her clients and to build rapport with others around her. Mary presented as anxious and had the tendency to take control during sessions with new clients or when faced with difficulty in session. Mary was very amicable, cooperative, and openly shared about her clients during supervision. She tended to rely less on her clinical intuition and more on structured methods such as worksheets. Mary responded well to verbal encouragement and when prompted she made the effort to challenge her clinical skills. Mary had an average of 20 clients per week ranging from children to adults. Her clients' presenting issues included depression, anxiety, substance use disorder, and adjustment disorder. Mary identified a desire to improve her Physical Self domain of the 5F-WEL and expected to (a) "have more energy," (b) "be happier when working with clients," and (c) "be more encouraging to clients to try something new because I have done that." She was compliant with the WELMS supervision plan and reported consistent engagement in her wellness activities.

\section{Study Setting}

The study was implemented at the counseling and training clinic (CTC) located at a Hispanic-serving institution in the Southern region of the United States, that offers free counseling services to members of the community across the lifespan within individual, couples, family, and group modalities. Counseling sessions were provided by counseling trainees in a private and confidential setting, and activities of the CTC were supervised by a licensed counseling faculty member and counselor education doctoral students. Access to counseling services was based on the academic calendar of the university.

\section{Intervention}

The WELMS was developed by Lenz and Smith (2010) and is based on the holistic, wellness-based approach towards client care as outlined in the Holistic Model for Treatment Planning (Myers, Sweeney, \& Witmer, 2000). The WELMS has two distinct qualities: (a) an overt emphasis on wellness education, appraisal, planning, and evaluation to promote supervisee skill development and client outcomes, and (b) a holistic, developmental perspective of supervisee lifestyle that supports engagement in career sustaining activities. Supervisory interventions were provided using the WELMS to promote development through four interrelated activities: Education, Assessment, Planning, and Evaluation (Lenz \& Smith, 2010). These activities were initially sequential, but became cyclical through the supervisory relationship.

The first step of the WELMS is education about the overall supervision model, identifying the supervisee's definition of personal wellness, reviewing formal models of wellness to be used during case conceptualizations, and explaining how improvement in wellness may contribute to the development of the supervisee's professional and personal life. Next, the WELMS involves an assessment of the supervisee's personal wellness using formal or informal assessments of wellness. Following, assessment results are used to develop an individual wellness plan for each supervisee based on the identified dimension that the supervisee deemed to be important to work on as they moved towards greater wellness and improved abilities as a counseling professional. Once the supervisee identified one specific dimension, the behavioral plan is developed by setting realistic and time-sensitive goals. Wellness plans include (a) an identified domain for monitoring and development, (b) a scaled satisfaction rating, (c) description of presumed ways that changes target domain may 
affect their personal and professional life, (d) related interventions, and (e) indications of progress (see Lenz \& Smith, 2010). Finally, supervisees were encouraged to commit to the supervision plan for at least four weeks and evaluated weekly during the wellness check-in aspect of the supervision sessions. After four weeks, supervisees were asked to report formative progress toward their goals and the wellness plan was modified as needed.

\section{Procedure}

Following institutional review board approval, CITs enrolled in the internship course, and obtaining internship hours at the CTC, were invited to participate in the study.

Baseline phase. Supervision with CITs was limited to case load management, case conceptualizations, and treatment planning from a cognitive behavioral therapy framework. Each week, the CITs completed the ProQOL assessment online via Qualtrics. Lengths of this baseline phase varied systematically between Diane (7 weeks), Susan (9 weeks), and Mary (11 weeks) to establish a multiple baseline paradigm for evaluation. Each participant completed the 5F-WEL assessment online at the end of the baseline phase and used the generated score report to develop an individualized wellness plan with the first author in preparation for beginning the WELMS.

Intervention Phase. Lengths of this intervention phase varied systematically between Diane (10 weeks), Susan (8 weeks), and Mary (5 weeks). CITs completed the ProQOL weekly and received one hour of weekly supervision for one hour, based on the WELMS and in reference to their individualized wellness plans. The first 15 minutes of supervision was dedicated to a wellness check-in and supporting CITs development and progress toward their wellness plans. The remaining 45 minutes focused on using the CBT framework, but also integrated holistic client conceptualization when identifying client issues. CITs maintained a weekly journal to document their experience receiving the WELMS. The journal prompts for each week were: (a) Describe your satisfaction with your wellness dimension this week, (b) In what ways did you improve on your wellness dimension this week? (c) How would you describe your professional quality of life this week? and (d) What was your experience of supervision this week?

\section{Data Analysis}

Participant ratings on the ProQOL during the baseline and treatment conditions were entered into Microsoft Excel files. Overall scores were calculated per the ProQOL manual, and visual depictions were generated for each participant according to the three subscales. Participant ratings for compassion satisfaction, burnout, and secondary trauma are situated on the vertical axis and time/phases on the horizontal axis. Course and efficacy of the WELMS were estimated across dependent variables using visual analysis and estimation of treatment effect.

Visual analysis. We completed the visual analysis of the ProQOL data by inspecting the differences in level, trend, and variability across the treatment phases (Kazdin, 2011). Changes in the level were deduced by comparing the mean for baseline to the treatment phase. A change in the mean across phases indicated the degree of change reported by the participant. The trend was evaluated by identifying the pattern of the data in the baseline and intervention phase, and drawing celebration lines using the split-middle line of progress. Variability was evaluated based on the range of scores within and between phases. The greater the degree variability in the treatment phase indicates less stability and a less defensible argument for the effectiveness of the intervention. By contrast, less variability indicates less vacillation and increased stability in behavior or performance and it can be stated that the intervention was effective (Lenz 2013).

Estimating Treatment Effect. The estimate of treatment effect was calculated using the Percentage of Data Exceeding the Median (PEM; Ma, 2006), which uses the median score in the baseline phase to determine the degree of nonoverlap between baseline and intervention data points (Vannest \& Ninci, 2015). Therefore, if the WELMS is efficacious, most data points will be either above the median point in the baseline for compassion satisfaction or below the median point in the baseline for burnout and secondary trauma. PEM was computed by: (1) identifying the median data point in the baseline phase, (2) using a ruler and pencil to draw a line through the 
data points in the baseline and treatment phases at the level of the median data point in the baseline, (3) counting the number of data points above or below the line in the treatment phase, (4) dividing the number of data points identified in step 3 by the total number of data points in the treatment phase (Lenz 2013; Parker, Vannest, \& Davis, 2011). PEM values range from 0 (0\%) to 1 (100\%) with each PEM score representing the treatment effect (Ma, 2006). We used interpretation guidelines by Scruggs and Mastropieri, (1998) to determine whether scores represented a very effective intervention (> 90\%), moderate effective intervention (70\%-89\%), debatable intervention (50\%-69\%), or ineffective $(<50 \%)$.

\section{Results}

Visual depictions of scores for secondary traumatic stress, compassion satisfaction, and burnout are presented in Figures 1, 2, and 3, respectively.

\section{Secondary Traumatic Stress}

Diane. Inspection of the mean level for Diane's baseline data indicated an average score of 14 on the secondary traumatic stress subscale of the ProQOL across seven weeks of measurement. By contrast, scores representing perceptions of secondary traumatic stress were slightly lower during the intervention phase $(M=13)$, suggesting a modest decrease of construct level over time. Evaluation of data trends between phases revealed a curvilinear, yet decreasing trend for ratings of secondary traumatic stress during the baseline phase, followed by an immediate and stable level of the data once beginning the WELMS intervention. Analysis of variability of data between phases revealed a 7-point range of scores during the baseline phase as opposed to a 3-point degree of variation during the intervention. The PEM estimate of treatment effect indicated that only $10 \%(\mathrm{PEM}=.10)$ of data points in the intervention phase were not overlapped with the baseline median (13) which is suggestive of an intervention that may not be effective. Taken together, these findings suggest that while the mean score for Diane's ratings on the secondary traumatic stress subscale decreased only slightly between the baseline and intervention phases, the reported effect showed an immediate and considerable degree stability within the therapeutic range of her reported values. However, the magnitude of treatment effect reported by Diane suggests these differences may not have had a practically significant effect on her experience of secondary traumatic stress during internship.

Susan. Analysis of the mean level for Susan's baseline data showed an average score of 24 on the secondary traumatic stress subscale of the ProQOL across nine weeks of measurement. However, scores representing secondary traumatic stress on the intervention phase were lower $(M=22)$, indicating a modest decrease of construct level over time. Inspection of data trends between phases revealed a curvilinear, yet decreasing trend for secondary traumatic stress scores during the baseline phase, followed by an immediate reduction and continued trend toward the desired effect among scores once the WELMS was implemented. Evaluation of variability of data between phases revealed a 6-point range of scores during the baseline and intervention phase. The PEM estimate of treatment effect indicated that $88 \%(\mathrm{PEM}=.88)$ of data points in the intervention phase were not overlapped with the baseline median (24), suggesting that the intervention may be moderately effective. Collectively, these findings suggest that while the mean score for Susan's ratings on the secondary traumatic stress subscale decreased slightly between the baseline and intervention phases, the reported effect showed an immediate reduction in her scores. The magnitude of treatment effect reported by Susan suggests these differences may have had a practically significant effect on her experience of secondary traumatic stress as an internship student.

Mary. Evaluation of the mean level for Mary's baseline data indicated an average score of 14 on the secondary traumatic stress subscale of the ProQOL across 11 weeks of measurement. By contrast, the scores representing secondary traumatic stress during the intervention phase were slightly lower $(M=13)$. This indicates

a modest decrease of construct level over time. Analysis of data trends between phases revealed a curvilinear, yet decreasing trend for ratings of secondary trauma, followed by an immediate and stable level of the data once the WELMS was executed. Inspection of variability of data between phases revealed a 5-point range of scores during 
the baseline phase as opposed to a 3-point degree of variation during the intervention phase. The PEM estimate of treatment effect indicated that $60 \%(\mathrm{PEM}=.60)$ of the data points in the intervention phase did not overlap with the baseline median (14). This suggests that the intervention may be within the debatably effective. Taken together, these findings suggest that while the mean score for Mary's ratings on the secondary traumatic stress subscale decreased slightly between the baseline and intervention phases, the reported effect showed an immediate reduction in her scores. The magnitude of treatment effect reported by Mary suggests these differences may have had a practically significant effect on her experience of secondary traumatic stress as an internship student.

\section{Compassion Satisfaction}

Diane. Inspection of the mean level for Diane's baseline data showed an average score of 39 on the compassion satisfaction subscale of the ProQOL across seven weeks of measurement. Compassion satisfaction scores on the intervention phase remained consistent $(M=39)$, indicating stability of the construct over time. Evaluation of data trends between phases revealed a linear trend for ratings of compassion satisfaction in the baseline phase, followed by an immediate and stable trend of data once the WELMS was implemented. Analysis of the variability of data between phases revealed a 7-point range of scores during the baseline phase. By contrast, variability during the intervention phase indicated a 2-point range of scores. The PEM estimate of treatment effect indicated that no data points $(0 \%$; $\mathrm{PEM}=0)$ in the intervention phase were not overlapped with the baseline median (40) which suggests that the intervention may be ineffective. Together, these findings suggest what while the mean score for Diane's ratings on the compassion satisfaction subscale did not increase between the baseline and intervention phases, the reported effect showed an immediate and considerable degree of stability within the therapeutic range of her reported values. However, the magnitude of treatment effect reported by Diane suggests these differences may not have had a practically significant effect on her experience of compassion satisfaction during internship.

Susan. Evaluation of the mean level for Susan's baseline data showed an average score of 38 on the compassion satisfaction subscale of the ProQOL across nine weeks of measurement. By contrast, the average score representing compassion satisfaction during the intervention phase was slightly higher $(M=39)$. Inspection of data trends between phases revealed a curvilinear trend for ratings of compassion satisfaction in the baseline phase, followed by an immediate and increasing trend of data once the WELMS was implemented. Analysis of the variability of data between phases revealed a 7-point range of scores during the baseline phase. By contrast, variability during the intervention phase indicated a 5-point range of scores. The PEM estimate of treatment effect indicated that $50 \%(\mathrm{PEM}=.50)$ of the data points in the intervention phase were not overlapped with the baseline median (38) which suggests that the intervention may be debatably effective. Collectively, these findings suggest that while the mean score for Susan's ratings on the compassion satisfaction subscale increased slightly between the baseline and intervention phases, the reported effect showed an immediate and considerable increase in her scores. However, the magnitude of treatment effect reported by Susan suggests these differences may have had a practically significant effect on her experience of compassion satisfaction during internship.

Mary. Analysis of the mean level for Mary's baseline data indicated an average score of 47 on the compassion satisfaction subscale of the ProQOL across 11 weeks of measurement. By contrast, the scores representing compassion satisfaction during the intervention phase were slightly lower $(M=46)$, indicating a slight decrease of the construct level over time. Evaluation of data trends between phases revealed a curvilinear, yet decreasing trend for ratings of compassion satisfaction, followed by an immediate reduction of the data once the WELMS was executed. Analysis of variability of data between phases revealed a 7-point range of scores during the baseline phase as opposed to a 6-point degree of variation during the intervention phase. The PEM estimate of treatment effect indicated that no data points $(0 \%$; $\mathrm{PEM}=0)$ in the intervention phase were not overlapped with the baseline median (48) which suggests that the intervention may be ineffective. Together, these findings suggest that the mean score for Mary's ratings on the compassion satisfaction subscale decreased slightly between the baseline and intervention phases, and the reported effect showed an immediate reduction in her 
scores. The magnitude of treatment effort reported by Mary suggests these differences may not have had a practically significant effect on her experience of compassion satisfaction as an internship student.

\section{Burnout}

Diane. Evaluation of the mean level for Diane's baseline data showed an average score of 20 on the burnout subscale of the ProQOL across seven weeks of measurement. However, scores representing burnout were slightly lower during the intervention phase $(M=19)$, suggesting a modest decrease of construct level over time. Analysis of data trends between phases indicated a curvilinear yet decreasing trend for ratings of burnout during the baseline phase, followed by an immediate curvilinear yet increasing trend once the WELMS was initiated. Inspection of variability between data phases revealed a 6-point range of scores during the baseline phase as opposed to a 4-point degree of variation during the intervention phase. The PEM estimate of treatment effect indicated that $50 \%(\mathrm{PEM}=.50)$ of the data points in the intervention phase were not overlapped with the baseline median (20), indicating that the intervention may be debatably effective. Taken together, these findings suggest that while the mean score for Diane's ratings on the burnout subscale decreased only slightly between the baseline and intervention phases, the reported effect showed an immediate degree of stability within the therapeutic range of her reported scores. However, the magnitude of treatment effect reported by Diane suggests these differences may have a practically significant effect on her experience of burnout.

Susan. Inspection of the mean level for Susan's baseline data indicated an average score of 24 on the burnout subscale of the ProQOL across nine weeks of measurement. Scores representing perceptions of burnout were lower during the intervention phase $(M=22)$. Combined, this suggests a modest decrease of construct level over time. Analysis of data trends between phases revealed a curvilinear yet increasing trend for ratings of burnout during the baseline phase, followed by an immediate decrease and decreasing trend of the data once the WELMS was implemented. Evaluation of variability of data between phases revealed a 5-point range of scores during the baseline phase as opposed to a 6-point degree of variation during the intervention. The PEM estimate of treatment effect indicated that $63 \%(\mathrm{PEM}=.63$ ) of data points in the intervention phase did not overlap with the baseline median (24), indicating that the intervention may be debatably effective. Collectively, these findings suggest that while the mean score for Susan's ratings on the burnout subscale moderately decreased between the baseline and intervention phases, the reported effect showed an immediate and significant reduction in her scores. The magnitude of treatment effect reported by Susan suggests these differences may have had a practically significant effect on her experience of burnout as an internship student.

Mary. Analysis of the mean level for Mary's baseline data indicated an average score of 19 on the burnout subscale of the ProQOL across 11 weeks of measurement. Burnout scores on the intervention phase remained consistent $(M=19)$, indicating stability of the construct over time. Inspection of data trends between phases indicated a curvilinear yet increasing trend for ratings of burnout, followed by an immediate reduction and decreasing trend of the data once the WELMS was initiated. Evaluation of variability of data between phases indicated a 6-point range of scores during the baseline phase as opposed to a 4-point degree of variability during the intervention phase. The PEM estimate of treatment effect indicated that $20 \%(\mathrm{PEM}=.20)$ of the data points in the intervention phase did not overlap with the baseline median (19). This suggests that the intervention may not be effective. Taken together, these findings suggest that while the mean score for Mary's ratings on the burnout subscale did not decrease between the baseline and intervention phases, the reported effect showed an immediate and considerable degree of stability within the therapeutic range of her reported values. However, the magnitude of treatment effect reported by Mary suggests these differences may not have had a practically significant effect on her experience of burnout as an internship student.

\section{Discussion}

This study provided some preliminary evidence associated with the efficacy of the WELMS for influencing aspects of professional quality of life with a sample of CITs completing internship requirements. In particular, the evaluation of the WELMS using a multiple baseline SCRD provided the impetus for inferences 
related to the utility of the intervention with respect to course and magnitude of effect and the importance of intervention dosage.

Results of this study provide evidence for the course of treatment of the WELMS for professional quality of life across compassion satisfaction, burnout, and secondary trauma. The course of treatment and trends appeared to be immediate across participants and across dependent variables. Evaluations of treatment effect, as evidenced by the PEM scores, were mixed but also appear to be a function of the interaction between dosage effect and time.

The findings for secondary traumatic stress depict trends for improvement, stabilization, and no improvement among secondary traumatic stress symptoms associated with 10, 8, and 5 weeks (respectively) of participation in the WELMS. It is probable that the CITs level of ability to self-regulate outside of supervision is a factor in their response to intervention. One explanation for this trend among scores relates to the interaction between dosage of intervention and degree of response. It is plausible that with greater exposure to the WELMS, participants were able to use supervision in a manner that mitigated the influence of client-CIT interactions on their professional quality of life. Inversely, minimal exposure to the intervention resulted in extended influence of secondary traumatic stress characteristics. Similar results were detected by Meany-Walen et al. (2016) who concluded CIT engagement in the WELMS was a protective factor for the experiences of increased risk for psychological stress during internship.

Results for compassion satisfaction reveal that the WELMS was debatably effective for increasing and stabilizing the degree of pleasure derived from working as a CIT. These findings depict trends for stabilization, improvement, and no improvement among compassion satisfaction symptoms associated with 10, 8, and 5 weeks (respectively) of participation in the WELMS. Similar to the trends for secondary traumatic stress, one explanation for this trend among scores is related to the interaction between dosage of intervention and the degree of response. It is possible that with increased time with the WELMS, participants were able to use supervision to help mitigate and stabilize the impact of client-CIT interactions and understand how self-compassion influences compassion satisfaction by having time to implement loving-kindness activities. Inversely, minimal exposure to the WELMS resulted in minimal mitigating effects. This is consistent with Storlie and Smith's (2012) findings that wellness-based interventions during supervision help to enhance the healthy development of CITs to be better prepared for the challenges of counseling others.

Findings for burnout suggests that the WELMS was effective for reducing and stabilizing the negative effects of caring for others. These findings depicted trends for no improvement associated with 10 weeks of participation in the WELMS and improvement associated with weeks 8 and 5 among burnout symptoms associated with participation in the WELMS. This trend among scores may be attributed to the timeliness of the intervention. It is plausible that when CITs are exposed to the intervention earlier in their training they were able to use supervision time in a manner that mitigated the effects of client-CIT interactions on their professional quality of life. This is especially important for those CITs with advanced greater distress who may not have taken the necessary steps to mitigate the effects prior to entering internship. One explanation for this trend among scores relates to the diverse personal stressors and complications related to degree completion; by contrast, earlier intervention resulted in maximized mitigating effects. This latter point is consistent with findings by Lenz et al. (2014) that CITs experienced greater resilience, well-being, and awareness about how their self-care translates into client care while of participating in the WELMS.

The degree of trauma-related cases varied across participants, in addition to clients who presented with difficult relational issues. These complex cases challenged the personal and professional development of the participants as it relates to their ability to control countertransference issues. Although there were diverse factors influencing the individual response, over time, to the WELMS, various factors influenced our analysis of the data including the use of multiple baseline design, direct observation of participants in their setting, and their entries for the journal prompts. For those participants who received 10 and 8 weeks of the WELMS, the intervention appears to be effective; however, for the one participant who did not receive a reasonable dosage of the WELMS, 
minimal effect was indicated which suggests that in addition to individual characteristics, having this intervention for less than 8 weeks is not as effective for treating issues related to secondary traumatic stress and compassion satisfaction. Although these results for the WELMS are promising, it is recommended that counselor supervisors consider unique student factors and the implementation of the WELMS to maximize intervention effectiveness.

\section{Recommendations for Counselor Supervisors}

Several recommendations for use of the WELMS with counseling students are available from the context of these findings within the context of professional literature. When considering that CITs are required to learn and demonstrate skills necessary for maintaining their professional quality of life, there is a body of evidence indicating the utility of integrating proactive approaches to develop career sustaining behaviors during the practicum and internship experiences (Lenz et al., 2012; Meany-Walen et al., 2016; Ohrt et al., 2015). Lawson (2007) found that in the absence of these skills, counselors are vulnerable to experiencing burnout and decreased clinical effectiveness. Therefore, it is important for supervisors to identify the baseline levels of distress and burnout of CITs with the understanding that practicum and internship activities may compound the effects of stressors over time. With this conceptualization of student experience, supervisors should implement the WELMS to mitigate negative influences on professional quality of life early during the relationship. Prompt introduction of the WELMS is justified by the noted pattern across their ProQOL variables wherein length of exposure during academic semester was associated with greater protective utility.

Furthermore, it is essential for supervisors to consider goodness of fit between the WELMS and important student characteristics including self-awareness, openness, and existing coping style. CITs with greater selfawareness regarding socioemotional processes during practice and internship placements may be better candidates for maximizing supervision experiences in general. However, when CITs are open to holistic conceptualizations of their development and proactive in understanding the many ways that individuals and groups can become well, professional quality of life may be a more achievable experience. Conversely, CITs who neglect to meet personal challenges with insight and openness may experience more countertransference and difficulty regulating emotions when under duress. For this reason, supervisors should help CITs develop WELMS interventions that are not only realistic and suitable to their needs, but also account for existing coping style. With a personalized approach to supervision and wellness engagement, CITs may progress through internship with a dynamic supportive framework to promote professional quality of life.

\section{Limitations of the Study and Recommendations for Future Research}

Although the results of this study provide some preliminary evidence of efficacy for the WELMS in relation to professional quality of life characteristics, some caveats are noted related to the study sample size, measurement of constructs, research method, and generalization of findings. Although the sample size for this study is sufficient to establish modest evidence for evidence-based practice, the results of three participants cannot be generalized to the population of CITs in CACREP programs. Furthermore, the participants and supervisor in this study were all women and therefore any inferences based on interactions between the WELMS and ProQOL scores may not be indicative of the experiences of men. Therefore, future SCRDs are needed with larger samples of diverse participants across multiple sites.

Additionally, our participants self-reported ProQOL data over 15 weeks, which may have increased the risk for them to self-report responses that are more desirable or respond in a manner they believed was anticipated. Future researchers are encouraged to collect data through varied approaches including more objective estimations that are less sensitive to test bias. Due to the nature of the setting, participants received supervision from multiple persons with varied clinical and supervisory experience at the CTC and in their assigned internship courses. Although each participant received an hour of the WELMS per week, participants also received supervision from multiple perspectives that may have obscured some of the true influence of the WELMS on ProQOL scores. Future researchers may consider having only one supervisor to work with participants to reduce the influence of these extraneous variables. Also, given the very controlled nature of the CTC setting, researchers are encouraged to investigate the usefulness of the WELMS in community and school settings where degrees of autonomy and oversight are disparate. 
Finally, we utilized multiple baselines to support inferences about causal association between the WELMS and ProQOL scores, yet researchers are encouraged to depict further treatment effects using different types of research designs such as between-groups and mixed methods studies. Adding a qualitative study with this design may help further explain and understand the lived experiences of students as they receive a wellness based model of supervision. In addition, implementing studies across the practicum and internship courses, such as extended multiple baseline designs or follow up studies (3, 6, and 12 months) may be beneficial to understanding the longitudinal impact of the WELMS.

\section{Conclusion}

Wellness based approaches to supervision can inspire CITs to become more proactive in their personal wellness and professional quality of life as supervisors help promote CIT skill development and career sustaining behaviors. However, few studies in which researchers have utilized a wellness model of supervision with CITs exist. Using an SCRD with multiple baselines, we found promising results for using the WELMS with CITs to help mitigate and stabilize client-CIT interactions on their professional quality of life. The results indicate that CITs who receive the WELMS are likely to show improvement in their professional quality of life, especially in the secondary traumatic stress and burnout subscales. The results also reveal the importance of using the WELMS in a time sensitive manner based on unique student needs.

\section{References}

American Counseling Association (2014). ACA Code of Ethics. Alexandria, VA: Author

Bernard, J. M., \& Goodyear, R. K. (2014). Fundamentals of clinical supervision. (5th ed.). Boston, MA: Pearson.

Bloomquist, K. R., Wood, L., Friedmeyer-Trainor, K., \& Kim, H. W. (2015). Self-care and professional quality of life: Predictive factors among MSW practitioners. Advances in Social Work, 16, 292-311. doi:10.18060/18760

Blount, A. J., \& Mullen, P. R. (2015). Development of an Integrative Wellness Model: Supervising counselors-intraining. The Professional Counselor, 5, 100-113.

Borders, L.D., Glosoff, H.L., Welfare, L.E., Hays, D.G., DeKruyf, L., Fernando, D.M., Page, B. (2014). Best Practices in Clinical Supervision: Evolution of a Counseling Specialty. The Clinical Supervisor, 33, 2644. doi:10.1080/07325223.2014.905225

Burck, A. M., Bruneau, L., Baker, L., \& Ellison, L. (2014). Emerging counselors’ perception of wellness: Implications for counselor development. Counseling Outcome Research and Evaluation, 5(1), 39-51. doi:10.1177/2150137813518554

Goodyear, R. K., Bunch, K., \& Claiborn, C. D. (2006). Current supervision scholarship in psychology. The Clinical Supervisor, 24, 137-147. doi:10.1300/j001v24n01_07

Kazdin, A. E. (2011). Single-case research designs: Methods for clinical and applied settings. (2nd ed.). New York, NY: Oxford University Press.

Lawson, G. (2007). Counselor wellness and impairment: A national survey. The Journal of Humanistic Counseling, Education and Development, 46, 20-34.

Lawson, G., \& Myers, J. E. (2011). Wellness, professional quality of life, and career-sustaining behaviors: What keeps us well? Journal of Counseling and Development, 89, 163-171

Lenz, A. S. (2013). Calculating effect size in single-case research: A comparison of nonoverlap methods. Measurement and Evaluation in Counseling and Development, 46, 64-73. doi:10.1177/0748175612456401

Lenz, A. S., Oliver, M., \& Sangganjanavanich, V. F. (2014). Perceptions of meaningful moments among counselors in training participating in the Wellness Model of Supervision. The Clinical Supervisor, 33, 45-62. doi:10.1080/07325223.2014.905814

Lenz, A. S., Sangganjanavanich, V. F., Balkin, R. S., Oliver, M., \& Smith, R. L. (2012). Wellness model of supervision: A comparative analysis. Counselor Education and Supervision, 51, 207-221. doi:10.1002/j.1556-6978.2012.00015.x 
Lenz, A. S., \& Smith, R. L. (2010). Integrating wellness concepts within a clinical supervision model. The Clinical Supervisor, 29, 228-245. doi:10.1080/07325223.2010.518511

Ma, H-H. (2006). An alternative method for quantitative synthesis of single-subject researches: Percentage of data points exceeding the median. Behavior Modification, 30, 598-617.

Meany-Walen, K. K., Davis-George, D., \& Lindo, N. A. (2016). The impact of wellness-focused supervision on mental health counseling practicum students. Journal of Counseling and Development, 94, 464-472. doi:10.1002/jcad.12105

Myers, J. E., \& Sweeney, T. J. (2005). The indivisible self: An evidence-based model of wellness (reprint). The Journal of Individual Psychology, 61, 269-279.

Myers, J. E., \& Sweeney, T. J. (2014). Five Factor Wellness Inventory. (2nd ed.). Palo Alto, CA: MindGarden.

Myers, J. E., Sweeny, T. J., \& Witmer, J. M. (2000). The wheel of wellness counseling for wellness: A holistic model for treatment planning. Journal of Counseling \& Development, 78, 251-266.

Neswald-Potter, R. E., Blackburn, S. A., \& Noel, J. J. (2013). Revealing the power of practitioner relationships: An action-driven inquiry of counselor wellness. Journal of Humanistic Counseling, 52, 177-190.

Ohrt, J. H., Prosek, E. A., Ener, E., \& Lindo, N. (2015). The effects of a group supervision intervention to promote wellness and prevent burnout. Journal of Humanistic Counseling, 54, 41-58. doi:10.1002/j.21611939.2015.00063.x

Parker, R. I., Vannest, K. J., \& Davis, J. L. (2011). Effect size in single-case research: A review of nine overlap techniques. Behavior Modification, 35, 303-322.

Perkins, E. B., \& Sprang, G. (2013). Results from the Pro-QOL-IV for substance abuse counselors working with offenders. International Journal of Mental Health and Addiction, 11, 199-213. doi:10.1007/s11469-0129412-3

Scruggs, T. E., \& Mastropieri, M. A., (1998). Summarizing single subject research: Issues and applications. Behavior Modification, 22, 221-242. doi:10.1177/01454455980223001

Stamm, B. H. (2010). The Concise ProQOL Manual. (2nd ed.). Pocatello, ID: ProQOL.org.

Storlie, C. A., \& Smith, C. K. (2012). The effects of a wellness intervention in supervision. The Clinical Supervisor, 32, 228-239. doi:10.1080/07325223.2013.732504

Thompson, E. H, Frick, M. H., \& Trice-Black, S. (2011). Counselor-in-training perceptions of supervision practices related to self-care burnout. The Professional Counselor: Research and Practice, 1, 152-162.

Vannest, K. J., \& Ninci, J. (2015). Evaluating intervention effects in single-case research designs. Journal of Counseling and Development, 93, 403-411. doi:10.1002/jcad.12038 
Figure 1: Secondary Traumatic Stress

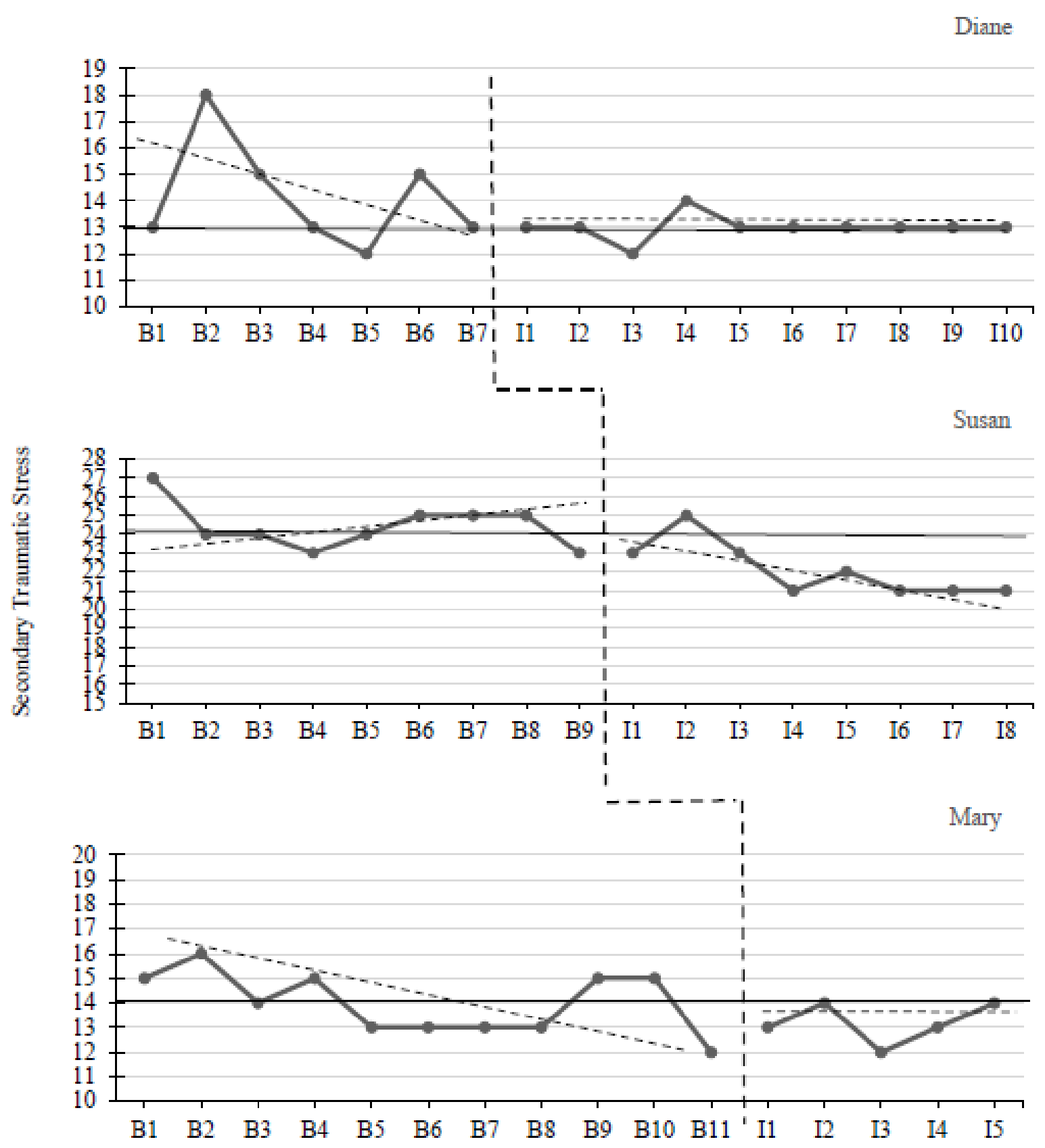

Figure 1. Visual depictions of participant scores on the secondary traumatic stress scale of the ProQOL. A decrease in scores is desirable. 
Figure 2. Compassion Satisfaction

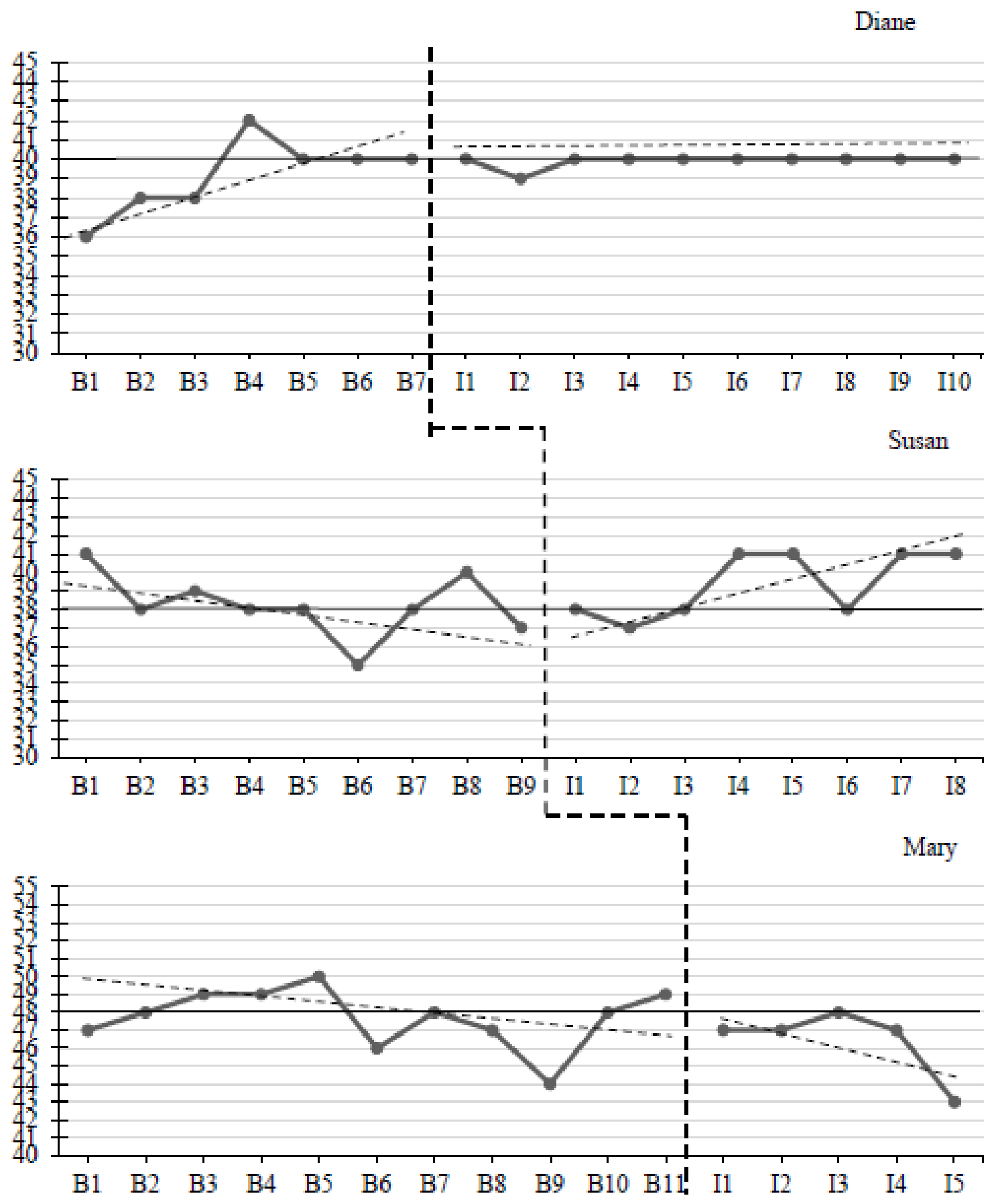

Figure 2. Visual depictions of participant scores on the compassion satisfaction scale of the ProQOL. An increase in scores is desirable. 
Figure 3. Burnout

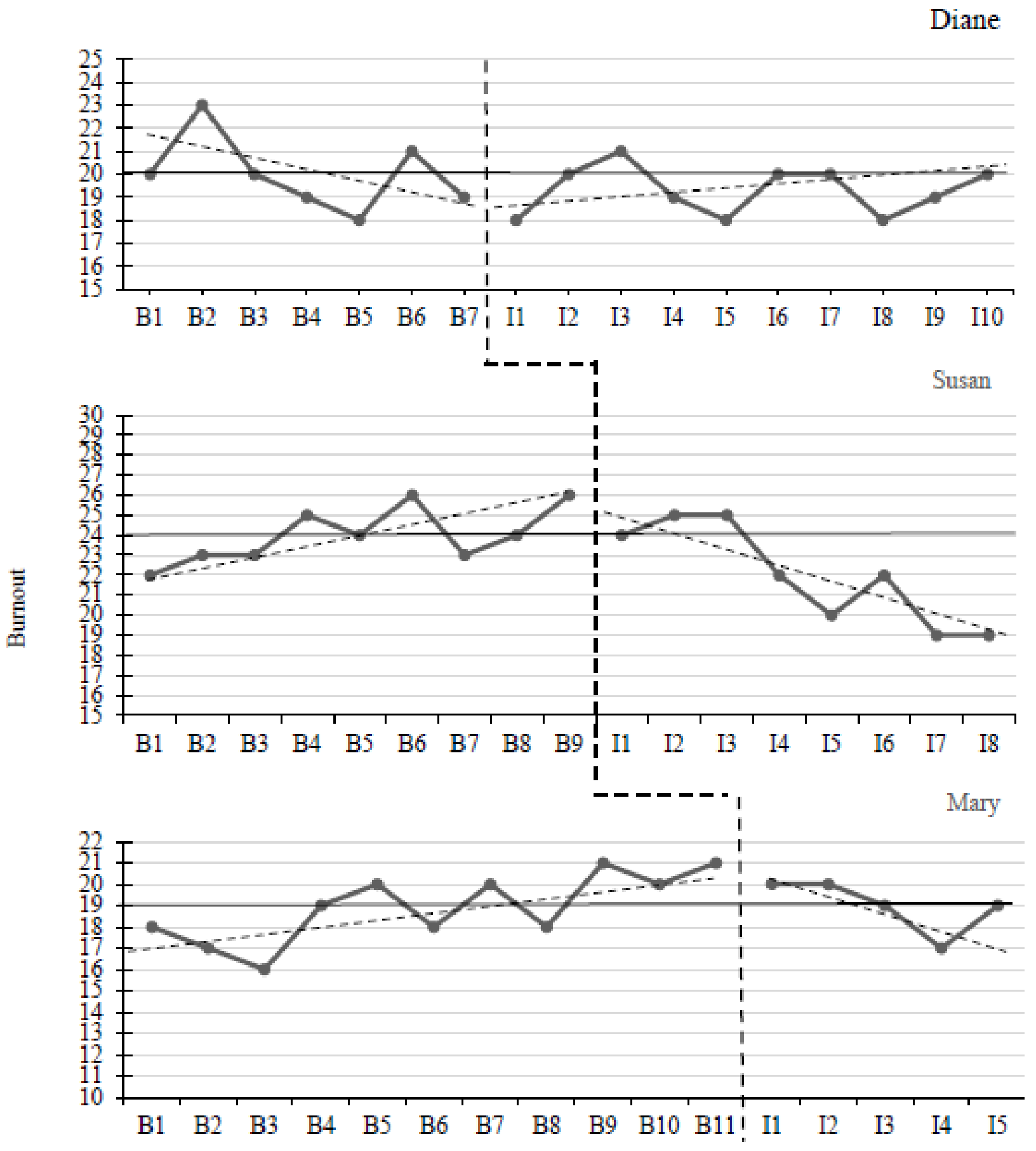

Figure 3. Visual depictions of participant scores on the burnout scale of the ProQOL. A decrease in scores is desirable. 